\title{
Histoire, Éducation, Innovation
}

\section{Pierre Boutan et Thierry Duclerc}

\section{OpenEdition}

Journals

Édition électronique

URL : http://journals.openedition.org/trema/1513

DOI : $10.4000 /$ trema. 1513

ISSN : 2107-0997

\section{Éditeur}

Faculté d'Éducation de l'université de Montpellier

\section{Édition imprimée}

Date de publication : 1 septembre 2003

Pagination : $1-5$

ISSN : 1167-315X

\section{Référence électronique}

Pierre Boutan et Thierry Duclerc, « Histoire, Éducation, Innovation », Tréma [En ligne], 22 | 2003, mis en ligne le 09 octobre 2012, consulté le 20 avril 2019. URL : http://journals.openedition.org/trema/1513 ; DOI : 10.4000/trema.1513

Ce document a été généré automatiquement le 20 avril 2019

Trema 


\title{
Histoire, Éducation, Innovation
}

\author{
Pierre Boutan et Thierry Duclerc
}

1 Ce numéro est né d'une boutade, à l'annonce de la préparation du numéro précédent de TREMA portant sur les innovations en matière éducative. C'était un moment où les IUFM, centres de formation initiale et continue des maitres, étaient constamment sollicités pour développer des changements immédiats dans le système éducatif. Avec une tendance à ne pas faire le retour en arrière nécessaire pour prendre la mesure du contexte: or l'histoire de l'éducation est abondamment pourvue de tentatives de changement bien plus souvent échouées que réussies. Par définition d'ailleurs, l'histoire, et l'histoire de l'éducation en général, n'existe-t-elle pas que par la description des changements qui sont intervenus dans son domaine?

2 Profitons-en, au passage, pour donner de "l'innovation" une définition suffisamment lâche: un changement dans le domaine éducatif destiné à lui apporter une amélioration; notions qui peuvent nous suffire, étant donné que les temps historiques qui nous concernent excluent pour l'essentiel les préoccupations contemporaines, redoutables, de l'administration de la preuve en matière de recherche. D'autant que les lexicographes ne nous fournissent pas d'autre information que celle d'un usage du mot d'abord dans le domaine économique (XVIII) puis technique (XIX)), son application au domaine éducatif datant de la deuxième moitié $d u X X^{e}$ siècle.

Compte tenu donc de l'existence à l'IUFM de Montpellier d'un Centre d'Études, de Documentation et de Recherche en Histoire de l'Éducation, il était naturel de solliciter des chercheurs d'horizons disciplinaires différents, mais tous préoccupés par l'histoire de l'éducation: Mathématiques ( $R$. d'Enfert), Physique (M. Guedj-Chauchard), Sciences du langage (P. Boutan), Sociologie (H. Terrai), STAPS (]. Gleyse, D. Denis), pour justement confronter leurs réflexions.

$4 \quad$ Faisons tout de suite les réserves nécessaires sur la portée des travaux qu'on va rencontrer. Personne ici ne veut donner à croire qu'il a fait le tour de la question traitée, au moins pour une raison simple : l'histoire de l'éducation est loin de disposer de tous les outils d'archives nécessaires à une vision précise de ce qui permet de prendre la mesure des changements. Prenons la base même de ce qui en est un élément central : les textes officiels. Leur recensement par le Service d'Histoire de l'Éducation de l'INRP est loin d'être mené à bien. Ainsi pour les disciplines scolaires, seuls les champs du français (A. Chervel), de l'histoire (P. Marchand), des sciences (B. Belhoste), des mathématiques (R. d'Enfert), commencent à être partiellement balisés. Paradoxe dans un pays centralisé comme la France, que de devoir faire des recherches pour recenser ces textes officiels! De 
toutes façons, et le paradoxe n'est pas moindre, il faut voir que leur déclinaison au niveau local est loin d'être négligeable, en particulier au niveau départemental, ce qui, dans le cas de l'enseignement $d u$ français par exemple, est une donnée très importante (voir l'article de P. Boutan).

Pour être la base de tout travail sur l'histoire des innovations, les instructions officielles ne peuvent constituer évidemment à elles seules un moyen suffisant : ainsi leur examen est le plus souvent associé au vaste champ des débats pédagogiques, comme l'illustre J. Gleyse. Mais aussi l'étude des manuels, intermédiaires si importants dans la plupart des disciplines, permet de mieux saisir les modulations de l'innovation demandée aux maîtres, entreprise amorcée ici par M. GuedjChauchard pour la physique du secondaire. Il faudrait bien sûr aller au-delà pour prendre la mesure de ce qui se passe réellement dans les classes, confronter rapports d'inspection, sentiment des maitres, des parents, des élèves, état de l'opinion; travail considérable, qu'il n'a pas été question bien sûr de pouvoir mener dans les domaines abordés ici. Cependant l'accès à des documents d'archives inédites, dans des conditions où le hasard a sa part, a servi de base aux contributions d'H. Terral et de P. Boutan.

6 Travaux d'autant plus nécessaires que l'image de l'histoire de l'éducation comme succession d'étapes homogènes de discours et de pratiques, voire, encore plus simpliste et pourtant fort répandue : opposant un passé traditionnel et détestable à un présent novateur et éclairé, est une perspective évidemment complètement fausse. Même si chaque époque peut avoir ses idées dominantes, le débat est l'état constant dans le champ éducatif, d'autant plus sans doute que les questions scolaires s'élargissent à toutes les couches et classes sociales. C'est clairement le cas à partir de la fin du XIXe siècle: on ne sera donc pas étonné de voir que tous les articles ici rassemblés traitent d'une période allant pour l'essentiel des années 1870 à la première guerre mondiale, soit la première partie de la Troisième République, qui mit en avant avec l'éclat que l'on sait sa volonté de répandre l'instruction en direction de tous ses enfants. Ce qui bien sûr n'empêche pas les auteurs de prendre en compte l'avant et l'après, sans négliger la période contemporaine, comme c'est le cas pour D. Denis ou $H$ Terrai.

$7 \quad$ L'échantillon des innovations examinées donne une idée de la diversité et en même temps de leurs points communs. Diversité des origines : l'innovation peut venir du haut, comme c'est le cas pour la rénovation du concept d'énergie dans l'enseignement secondaire (M. Guedj-Chauchard) ou la différenciation de l'enseignement de la géométrie entre secondaire et primaire (R. d'Enfert). Elle peut aussi faire l'objet d'une démarche venue de maitres eux-mêmes, avec l'ambition de servir un engagement militant (ainsi les félibres méridionaux, le frère Savinian et Perbosc). Enfin c'est une perspective historique plus vaste que choisit J. Gleyse, étendant à toute la discipline de l'«EPS », le champ de ses considérations. D. Denis va au-delà encore, pour s'intéresser à la forme scolaire ellemême dans ses rapports avec l'extrascolaire justement, explorant l'hypothèse que le défaut de réussite dans le changement propre au domaine scolaire peut expliquer que l'on ait tenté de produire ce changement par l'extérieur, en particulier par le scoutisme, conçu d'abord sur un modèle militaire.

8 Ce qui nous amène à mettre en avant un autre des points communs à toutes ces études: aucune ne peut abstraire l'innovation envisagée de son contexte idéologique au sens large, qu'il soit social ( $R$. d'Enfert), épistémologique (M. Guedj-Chauchard), politique (J. Gleyse, P. Boutan, H. Terrai, D. Denis). C'est bien constater que la lutte des idées est toujours présente, et de plus reliée de façon plus ou moins directe à notre actualité. Ce qui ne veut évidemment pas dire que l'histoire des innovations est semblable au mythe de l'éternel retour, mais qu'à l'ignorer, on s'expose pour le moins à perdre du temps. 
Justement parce que le recul nous donne la possibilité de mieux voir, et sans doute de mieux comprendre, comment une innovation peut réussir ou échouer. Parmi les intervenants, il y a ceux qui s'interrogent sur la permanence de ce qui réussit, à être majoritaire au moins, et de ce qui parait devoir être toujours minoritaire; de ce point de vue le fait d'être du côté du pouvoir hiérarchique, s'il constitue un avantage indéniable, n'est pas pour autant suffisant pour assurer le succès, mais la prise de positions "en haut" devient un enjeu décisif, qui peut nécessiter des dizaines d'années d'efforts. Dans l'échantillon des innovations ici considérées, on trouve des réussites sans doute, et aussi des échecs, du moins dans la période considérée, mais avec le sentiment qu'une époque plus contemporaine reprendra ce qui auparavant avait échoué ( $P$. Bouton, H. Terral).

Le lien avec l'histoire des idées montre à l'évidence la relation entre les valeurs dominantes et la réussite des innovations à une époque donnée. Mais étant donné l'hétérogénéité de la société, rien n'est facilement acquis en terme de changements en profondeur, si les acteurs ne sont pas convaincus de l'intérêt de ces changements. La résistance est régulièrement l'indice de débats, d'où l'importance de disposer de soutiens, en particulier dans les vecteurs d'opinion que sont à l'époque les seuls journaux, dont la diversité d'opinion est sans commune mesure avec aujourd'hui. La reconnaissance du prestige d'une opinion, associée à un homme ou à une idée, voilà un facteur de poids, même si on peut toujours lui opposer d'autres hommes et d'autres idées. Ainsi, au nom du Progrès que tout le monde affirme vouloir poursuivre, on peut chercher à convaincre: mais encore faut-il vérifier si ce progrès est aussi réel qu'il y paraît, et si l'effort nécessaire au changement en vaut finalement la peine.

11 Pas d'innovation vraiment sérieuse en effet sans effort à faire par le plus grand nombre des acteurs : les élèves bien entendu, si le changement demandé est immédiat, et remet en cause ce qui était en principe au moins déjà appris, ce qui n'est pas un cas si fréquent. Mais surtout il faut convaincre ceux qui jugent des résultats: les parents, qui sont aussi les électeurs (au moins la moitié masculine jusqu'en 1945), surtout les maitres au bout du compte, qu'il faut persuader dans leur masse, au-delà d'une élite active mais dont le nombre réduit ne peut suffire pour emporter l'adhésion, dans une époque où la diffusion des innovations a besoin pour être efficace du relais de la hiérarchie officielle, quand elles viennent d'en bas. Condition nécessaire mais pas suffisante. Consentir des efforts, c'est passer par un bilan comptable: que rapporte l'innovation, en terme de satisfaction pour le maitre; que peut-il, que va-t-il gagner à faire des efforts? Contre la hiérarchie, on n'a de chances que détrouver des militants pour faire face, et cela fait numériquement peu. Avec la hiérarchie, c'est selon: ou l'on voit ce qu'on peut gagner en terme de considération dans le jugement de l'inspection, ce qui est fait de la réussite des élèves, à la limite considération du devoir accompli au regard de l'amour qu'on porte à la Vérité, à Dieu, au Peuple, à la Patrie. Ou l'on se réfugie dans un attentisme que l'expérience a permis de conforter: les textes officiels sont une chose, la tranquillité professionnelle en est une autre, sans que le temps qui passe démontre toujours que l'on avait tort de ne rien changer, puisque de nouvelles instructions officielles peuvent revenir sur leurs prescriptions précédentes: situation qui tend à s'accélérer dans la deuxième moitié du XXe siècle.

12 Encore un biais enfin pour se débarrasser du problème: l'inspecteur dit de changer ceci ou cela, mais c'était déjà fait... On rencontrera ici de ces ruses, que Michel de Certeau a justement évoquées ${ }^{1}$

13 C'est dire que l'intérêt pour l'innovation est multiforme, et ne se résume pas à l'enthousiasme spontané que, selon ses thuriféraires naïfs, il devrait susciter : la perspective historique montre en tout cas que ce n'est pas toujours sans justification. 


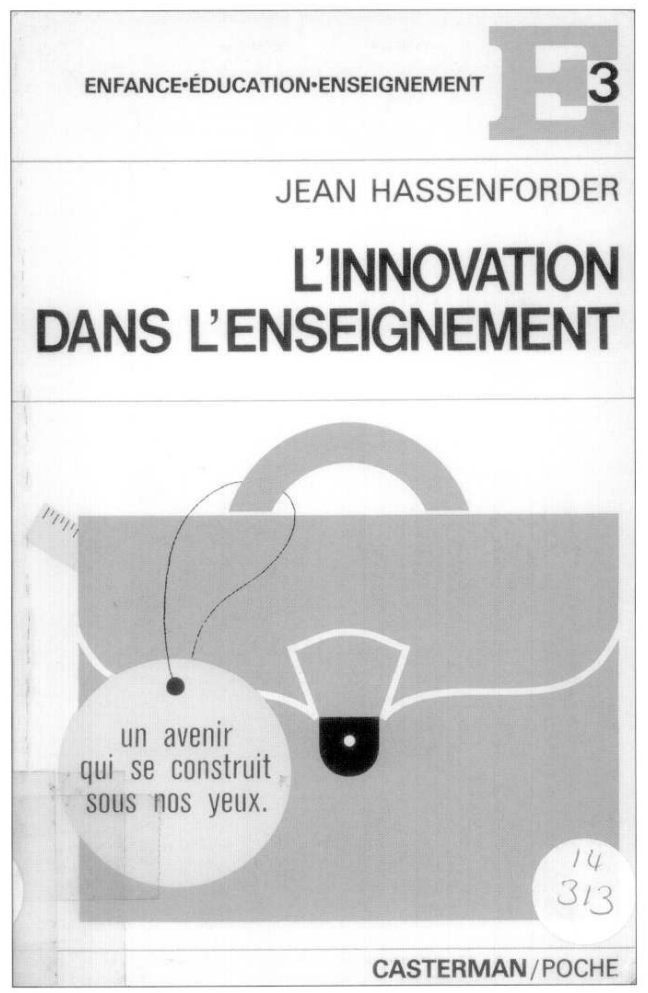

Un des premiers ouvrages sur l'innovation en pédagogie.

HASSENFORDER J. : L'INNOVATION DANS L'ENSEIgNEMENT. Tournai (Belgique), Casterman-Poche (collection : E3), 1972.

\section{NOTES}

1. CERTEAU Michel de : L'invention du quotidien I Arts de faire.... Paris, Gallimard, 1990.

\section{INDEX}

Mots-clés : histoire, éducation, innovation

Keywords : history, education, innovation 